\title{
Gestión de la calidad educativa en educación superior del sector privado
}

Quality Management in Private Higher Education

Management de la qualité éducative dans l'éducation supérieure du secteur privé Gestão da qualidade da educação no ensino superior-setor privado

Fecha de recepción: 15 DE JUNIO DE 2012/ Fecha de aceptación: 10 DE DICIEMBRE DE 2013

Encuentre este artículo en http://magisinvestigacioneducacion.javeriana.edu.co/

doi:10.11144/Javeriana.M6-13.GCEE

Escrito por Martha Armida Fabela-Cárdenas Universidad Autónoma de Nuevo León MONTERREY, MÉXICO mfabela36@hotmail.com

Alfonso Hernán García-Treviño Universidad Autónoma de Nuevo León MonterRey, MÉxICO agarciat@prodigy.net.mx

\section{Resumen}

Este artículo presenta la teoría emergente sobre los factores determinantes para ofrecer educación de calidad en instituciones de educación superior (IES) del sector privado. En particular, se presenta un modelo de factores y subfactores que influyen en la calidad de la educación y generan éxito y que emerge como resultado de entrevistas a profundidad realizadas con líderes de dichas instituciones educativas. El estudio se llevó a cabo con una muestra de veintidós líderes de instituciones de educación superior del sector privado, con una metodología Delphi o consulta a expertos y revela una actualización del modelo de Arcaro de 1995.

\section{Palabras clave autor}

Modelos de control de calidad en educación, gestión de la calidad, factores de éxito, liderazgo.

\section{Palabras clave descriptor}

Calidad de la educación-modelos de evaluación, educación superior, administración de la calidad, éxito, liderazgo.

\section{Transferencia a la práctica}

Los resultados que aquí se muestran pueden servir como guía para todos los gestores y actores en educación interesados en conocer los aspectos que se toman en cuenta en los modelos de gestión de la calidad en educación, originados en los procesos de aseguramiento de la calidad del sector empresarial, para mantenerse con éxito en el contexto de educación superior del sector privado en México.

Para citar este artículo / To cite this article / Pour citer cet article / Para citar este artigo

Fabela-Cárdenas, M. A., \& García-Treviño, A. H. (2014). Gestión de la calidad educativa en educación superior del sector privado. magis, Revista Internacional de Investigación en Educación, 6 (13), 65-82. 


\section{Key words author}

Models of Quality Control in Education, Quality Management, Success Factors, Leadership.

\section{Key words plus}

Educational Quality-evaluation Models, Education Higher, Quality Control, Success, Leadership.

\section{Abstract}

This paper puts forward emerging theory on determining factors for quality of education in private higher education institutions. A model of factors and sub-factors that influence the quality of education, result of in-depth interviews with leading figures of the institutions, is presented. The study was conducted with a sample of twenty-two leading figures of private higher education institutions, using a Delphi methodology or expert consultation, and it reveals an update of Arcaro's model of 1995
Transference to practice

The results described in this paper may serve as a guide to those interested in getting to know the characteristics of quality management models in education. These models are based on corporate processes of quality management, and allow institutions to successfully be part of the Mexican private higher education system.

\section{Mots clés auteur}

Modèles de maîtrise de la qualité dans l'éducation, management de qualité, facteurs de succès, leadership.

\section{Mots clés descripteur}

Qualité de l'éducation-modèles d'évaluation, enseignement supérieur, qualitécontrôle, succès, leadership en éducation.

\section{Résumé}

Cet article présente la théorie émergeante par rapport aux facteurs déterminés pour offrir éducation de haute qualité dans les institutions d'éducation supérieure (IES) du secteur privé. Notamment, on présente un modèle de facteurs et subfacteurs qui ont une influence dans la qualité de l'éducation et produisent du succès et émergent en tant que résultat d'entretiens exhaustifs réalisés aux leaders des institutions éducatives. L'étude a été menée avec une démonstration de vingt-deux leaders d'institutions d'éducation supérieure du secteur privé, avec une méthodologie Delphi à experts et montre une actualisation du modèle Arcaro de 1995.

\section{Transfert à la pratique}

Les résultats qu'on montre ici peuvent être utiles en tant que guide pour tous les managers et acteurs en éducation intéressés pour connaître les aspects qui sont prises en compte dans les modèles de management de la qualité en éducation, crées dans les processus d'assurance de la qualité du secteur des entreprises, pour se garder avec succès dans le contexte de l'éducation supérieure du secteur privé au Mexique.

\section{Palavras-chave autor}

Modelos de controle de qualidade na educação, gestão da qualidade, fatores de sucesso, liderança.

\section{Palavras-chave descritor}

Gestão da qualidade total na educação, ensino secundário, controle de qualidade, exito, liderança.

\section{Resumo}

Este artigo apresenta os fatores que determinam a oferta de uma educação de qualidade nas instituições de ensino superior (IES) do setor privado. Em particular, apresenta um modelo de fatores e subfatores que influencia na qualidade da educação gera sucesso e surge como resultado de entrevistas em profundidade realizadas com líderes dessas instituições de ensino. 0 estudo foi realizado com uma amostra de vinte e dois dirigentes de instituições, com a metodologia Delphi, ou consulta a peritos, e revela uma atualização do modelo Arcaro 1995.

\section{Transferência à prática}

Os resultados aqui apresentados podem servir como guia para todos os gestores e agentes da educação interessados em compreender os aspectos mais relevantes nos modelos de gestão de qualidade, originados nos processos de garantia de qualidade no setor empresarial, com o fim de manter o sucesso no contexto do ensino superior no setor privado no México. 


\section{Introducción}

Con la apertura de fronteras, los tratados de libre comercio y la creación de bloques económicos que propiciaron la formación de mercados globales, tanto México como el resto de los países del mundo entraron en la competencia con base en la calidad de los bienes y servicios que ofrecen. Esta pugna ha generado un gran movimiento orientado a diseñar sistemas que aseguren la calidad de los bienes o servicios que cada Nación ofrece para consumo interno o externo.

Tal necesidad de asegurar la calidad de bienes y servicios se apoyó en la filosofía y los procesos del sistema de gestión de la calidad. De esta manera, el comercio global inició un gran movimiento para establecer dichos sistemas, que no solo abarca a las empresas del sector privado, sino que se ha extendido a todos los ramos que componen la economía de un país y que recientemente ha aparecido en el ámbito educativo. En la actualidad es característico constatar que los rectores de las diferentes universidades se interesan por encontrar nuevas formas para mejorar los servicios educativos que ofrecen a la comunidad, tras observar si estos son de calidad.

El propósito de este artículo es mostrar los resultados de un estudio que indaga sobre los posibles factores que influyen en el éxito de las instituciones de educación superior del sector privado, con base en la calidad educativa desde la perspectiva de sus líderes. Los hallazgos presentados pueden orientar a otros actores de la educación que se interesen por conocer los aspectos que se toman en cuenta para mantenerse con éxito y con calidad en un contexto cada vez más competitivo. En este artículo se presenta la teoría emergente sobre los factores que influyen en la gestión de la calidad educativa, por medio de un análisis cualitativo de las percepciones de veintidós líderes de instituciones de educación superior del sector privado en México.

\section{Referentes conceptuales}

\section{Calidad en la educación superior}

Este estudio se adhiere a los conceptos de calidad educativa definidos por la Unesco, según la cual debe ser equitativa, relevante, pertinente, eficaz y eficiente. La Unesco asume que, como organismo, su papel frente a la educación superior es:

[...] proponer instrumentos y una síntesis de las prácticas idóneas a fin de ayudar a los Estados Miembros a evaluar la calidad y pertinencia de la educación superior transfronteriza y proteger a los estudiantes y demás partes interesadas contra una educación superior de mala calidad (Unesco, 2006, p. 5).

La Unesco (2006) propone directrices como punto de partida para garantizar la calidad de la educación superior y al mismo tiempo, impulsa el aumento de capacidades nacionales y regionales para mejorar los dispositivos de garantía de calidad y los mecanismos de acreditación (Unesco, 2008).

En suma, la calidad educativa se define como la instrucción académica superior impartida en su conjunto teórico-práctico, que permite a los graduados responder a las exigencias de su profesión y, a la vez, incorporarse al mercado laboral o continuar sus estudios de especialidad o posgrado, cuando los programas cursados con anterioridad en la IES de procedencia son reconocidos y validados. Una institución que brinda una
Descripción del artículo | Article description | Description de l'article | Artigo descrição

Este artículo de investigación se deriva del proyecto "Factores clave de éxito en la gestión de calidad educativa en las instituciones de educación superior privadas del estado de Nuevo León", desarrollado en el posgrado de la Facultad de Filosofía y Letras de la Universidad Autónoma de Nuevo León. 
educación de calidad consigue una imagen de respeto y aceptación de sus egresados por parte de la sociedad, su entorno económico, sus instituciones y otros centros educativos de nivel superior.

Lograr que todos los individuos accedan a una educación superior de calidad, que les permita dominar los conocimientos indispensables para desenvolverse competitivamente en el desempeño de su profesión, es uno de los retos que exige inmediata atención. Si la función de la educación es el desarrollo continuo de la persona, se debe reflexionar sobre el papel asignado a las instituciones educativas como encargadas de formar a las nuevas generaciones de profesionales.

\section{La privatización de la educación superior}

Respecto al acceso a una educación superior de calidad, Laval (2004) afirma que la concepción del neoliberalismo sobre la escuela es la de un mercado competitivo en el que "las empresas o cuasi empresas especializadas en la producción de servicios educativos pretender satisfacer las necesidades de los individuos con el suministro de mercancías o cuasi mercancías" (p. 135). De acuerdo con Laval, esta concepción neoliberal de la educación deja de lado la noción de la educación como una lógica política para favorecer la igualdad, la solidaridad o la redistribución en un territorio nacional. El mismo autor afirma que las ideas de hacer de la escuela una empresa se incorporaron al discurso oficial, en particular, al justificar reformas educativas (en muchas Naciones, no solo en Estados Unidos, en donde se originan). Este argumento se incorpora también al de los actores y administradores de la educación. Laval (2004) explica que:

Esta imitación retórica y esta interiorización subjetiva acabaron por imponerse en una escala social más amplia hasta el punto de que las propias fuerzas que habrían podido y debido oponerse a ellas entraron en ocasiones en una lógica de emulación (p. 268).

Las consecuencias que tiene esta tendencia de privatización de la educación, según Laval (2004), es que las familias con mejores medios económicos salen beneficiadas con esta política de la libre elección de escuela y arguye que:

[...] el impulso a favor de la privatización se explica en gran parte por el deterioro de la escuela pública, en lo que respecta tanto a las condiciones materiales como a las deplorables condiciones pedagógicas que reinan en ella en ciertos casos (p. 139).

También advierte que estas condiciones ocasionarán que los estudiantes con mejor posición económica abandonen la escuela pública por la privada y generen así la constitución de escuelas-guetos que favorecen al sector privado.

Si bien hay un debate sobre el derecho a la educación gratuita y de calidad en México y en América Latina, se reconoce que existen instituciones de educación del sector privado que subsisten porque han alcanzado cierto prestigio en la sociedad y porque a todas luces hay estudiantes interesados en asistir a dichos centros educativos, a pesar de sus costos de matrícula. Este artículo no pretende justificar la educación privada, sino analizar las maneras en que las instituciones de educación superior del sector privado conquistan el éxito.

\section{La evaluación de la calidad en la educación superior}

La tendencia para evaluar la calidad de la educación con base en los procesos de aseguramiento de la calidad en las empresas ha tenido influencia en México. Estas tendencias se revelan cuando se incorporan dichos procesos en la evaluación de instituciones de educación del sector público y del sector privado, al seguir, por ejemplo, procesos de certificación como el ISO 9000, de origen empresarial. En México, las instituciones de educación superior buscan ser acreditadas por dichos procesos de certificación. Véase, como prueba, el sitio web de la Asociación Nacional de Universidades de Educación Superior (ANUIES) y una lista de instituciones mexicanas acreditadas por ISO 9000.

Por supuesto, hay varios modelos para la evaluación educativa con base en los modelos de calidad empresarial (Peck \& Reitzug, 2012), entre ellos, el modelo Baldridge, una lista de criterios que proporciona una estructura integral para evaluar instituciones educativas (Furst-Bowe \& Bauer, 2007). De acuerdo con FurstBowe y Bauer, el modelo surgió primero para evaluar la competitividad de compañías manufactureras en Estados Unidos y luego fue revisado y modificado para organizaciones educativas y de la salud a mediados de la década del noventa. Los criterios se enfocan en siete categorías: Liderazgo, Planificación Estratégica; Estudiantes, inversionistas y mercado; Medición, análisis y manejo del conocimiento; Profesorado y personal; Manejo de procesos; y Resultados de desarrollo organizacional (Furst-Bowe \& Bauer, 2007).

Otro modelo, este de origen europeo, de la Fundación Europea para el Manejo de la Calidad (EFQM, por su nombre en inglés) es el conocido como el EFQM Excellence Model Higher Education Version $2003^{\circledR}$, adaptado para educación superior por la universidad británica Sheffield Hallam University. Tiene ocho criterios de guía: Enfoque en el cliente; Orientación hacia resultados; Liderazgo y propósito; Participación y desarrollo humano; Manejo de procesos y datos; Desarrollo de socios; Responsabilidad social corporativa; y Aprendizaje continuo, innovación y mejora (Steed, Maslow 
\& Mazaletskaya, 2005). Dado que el tema es controversial, este modelo tiene detractores que consideran que dichos modelos de evaluación tienen deficiencias conceptuales, que no pueden enfrentar las particularidades de la educación superior y que son modas destinadas a desaparecer (Temple, 2005) o modas que se repiten en ciclos (Peck \& Reitzug, 2012).

A pesar de las críticas, es evidente que hay interés en evaluar la educación superior con modelos de base empresarial y en el contexto mexicano también se observa esta tendencia. Este estudio adopta como modelo para el análisis de los resultados de la investigación el modelo de Arcaro (1995), porque tiene como base la literatura clásica sobre la gestión de la calidad en las empresas. El modelo de Arcaro no es el único disponible y puede ser perfectible, aunque tiene criterios similares con otros modelos.

Comparación de criterios entre tres modelos de calidad

En la Tabla 1 se ofrece una comparación entre los criterios de tres modelos: Baldridge (Furst-Bowe \& Bauer, 2007), EFQM Excellence Model (Steed, Maslow \& Mazaletskaya, 2005) y de Arcaro (Arcaro, 1995).

Tabla 1.

Comparación de criterios entre tres modelos de evaluación en educación

\begin{tabular}{llll}
\hline $\begin{array}{l}\text { Modelo Baldridge } \\
\text { (Furst-Bowe \& Bauer, }\end{array}$ & $\begin{array}{l}\text { Modelo } \\
\text { EFQM Excellence Model } \\
\text { (Steed, Maslow \& Mazaletskaya, 2005) }\end{array}$ & $\begin{array}{l}\text { Modelo de Arcaro } \\
\text { (Arcaro, 1995) }\end{array}$ \\
\hline Criterios & $\begin{array}{l}\text { Planificación estratégica } \\
\text { Liderazgo }\end{array}$ & Liderazgo y propósito & $\begin{array}{l}\text { Planificación estratégica (mercados, } \\
\text { necesidades, metas y objetivos) }\end{array}$ \\
\hline $\begin{array}{l}\text { Profesorado y personal } \\
\text { Manejo de procesos }\end{array}$ & Participación y desarrollo humano & $\begin{array}{l}\text { Comunicación (de metas y objetivos) } \\
\text { (elegir docentes, directivos y medidas } \\
\text { de desempeño) }\end{array}$ \\
\hline $\begin{array}{l}\text { Estudiantes, } \\
\text { inversionistas y mercado }\end{array}$ & Desarrollo de vínculos o vinculación & $\begin{array}{l}\text { Implementación del PE (participación } \\
\text { de equipos de trabajo) }\end{array}$ \\
\hline $\begin{array}{l}\text { Medición, análisis y } \\
\text { manejo del conocimiento procesos y datos (facts) }\end{array}$ & Responsabilidad social corporativa & $\begin{array}{l}\text { Medidas de desempeño (del programa } \\
\text { y del entrenamiento) }\end{array}$ \\
\hline $\begin{array}{l}\text { Resultados de desarrollo conflictos } \\
\text { organizacional }\end{array}$ & Orientación hacia resultados & $\begin{array}{l}\text { Validación del PE (medición de } \\
\text { resultados, modificar PE, documentar } \\
\text { modificaciones) }\end{array}$ \\
\hline \hline
\end{tabular}

Fuente: elaboración propia

Esta comparación muestra que los tres modelos coinciden exactamente en cinco criterios: Liderazgo; Profesorado y personal; Manejo de procesos; Medición; y Resultados. El modelo EFQM no tiene el componente de Planificación estratégica, pero agrega Responsabilidad social corporativa. El modelo Baldridge no tiene el componente Mejora continua. El modelo de Arcaro tiene siete componentes, aunque pudiera mejorarse si se le agregara el componente de Responsabilidad social corporativa.

De acuerdo con Arcaro (1995), los principios de gestión de la calidad aplicados con éxito en las empresas también pueden ser aplicados en las 
instituciones educativas, lo cual requerirá compromiso, tiempo, perseverancia, cambio de actitud e inversión en capacitación de todos los miembros de una institución. Con frecuencia, se puede pensar que la calidad educativa solo mejoraría si la sociedad inyectara más dinero en el sistema educativo, pero según Arcaro (1995), el dinero no es la única clave para ello, puesto que es preciso que el personal administrativo, los maestros, los directivos y el consejo de una institución educativa desarrollen nuevas actitudes que se enfoquen al liderazgo, al trabajo en equipo, a la cooperación y al reconocimiento de los sistemas de calidad como una nueva filosofía de trabajo (Arcaro, 1995).

\section{El concepto de calidad empresarial}

El concepto de sistema de calidad viene de Deming (1994), cuando indica que el sistema, en cuanto institución, empieza con: a) una predicción de la gerencia relativa al mercado que podrá tener el producto o servicio en el futuro; b) se continúa con el diseño del producto o servicio; c) se define el abastecimiento de equipo y materiales, la manufactura, la prueba, el mercadeo del producto o servicio; d) se investiga el mercado, y e) se rediseña; así en un ciclo interminable.

El creador de la idea de liderazgo en la promoción del sistema de calidad viene de Juran (2000), quien reconoce la importancia del líder en las funciones que le corresponden en la promoción del control de calidad. A partir de aquí se reconoció tal control como un instrumento de la gerencia que más tarde se llamó gestión de la calidad. De acuerdo con Ishikawa (1986), Total Quality Control (TQC) o gestión de la calidad fue un concepto desarrollado por Feigenbaum en 1956 y fue definido como un sistema eficaz para integrar los esfuerzos en materia de desarrollo, mantenimiento y mejoramiento de la calidad realizados por los diversos grupos de una organización, de modo que fuera posible producir bienes y servicios más económicos y compatibles con la plena satisfacción de los clientes.

Existen varios autores que tratan el tema de la gestión de la calidad educativa; sin embargo, con el fin de dar un marco de referencia a este estudio, se seleccionó a Jerome S. Arcaro (1995), porque se considera que, dentro del marco educativo, desarrolla y propone un sistema de gestión de calidad bien estructurado que permite el análisis de los temas existentes y que sirve de base para indagar si hay temas nuevos o emergentes.

Jerome S. Arcaro (1995) adopta esta filosofía de gestión de la calidad; propone que por medio de este sistema los profesionales de la educación ayuden a sus alumnos a potenciar las habilidades que necesitarán para competir en una economía ya globalizada. También reconoce que los sistemas de gestión de la calidad desarrollados para las empresas no deben de ser adoptados literalmente para su aplicación en instituciones educativas, aunque admite que muchos de sus principios pueden ser ajustados para crear un propio modelo de gestión de la calidad. Arcaro (1995) recoge ciertos principios de Deming (1994) que tienen aplicación en instituciones educativas:

a) La directiva y la Administración de las instituciones educativas deben aceptar la meta de calidad como un objetivo educativo.

b) El énfasis debe estar más en la prevención, que en la detección de los fracasos educativos de los estudiantes.

c) El uso de métodos de control estadístico debe implementarse para ayudar a la gestión administrativa de la institución, así como para mejorar el desempeño de los estudiantes. 
Figura 1.

Pilares básicos para la gestión de la calidad educativa de J. S. Arcaro

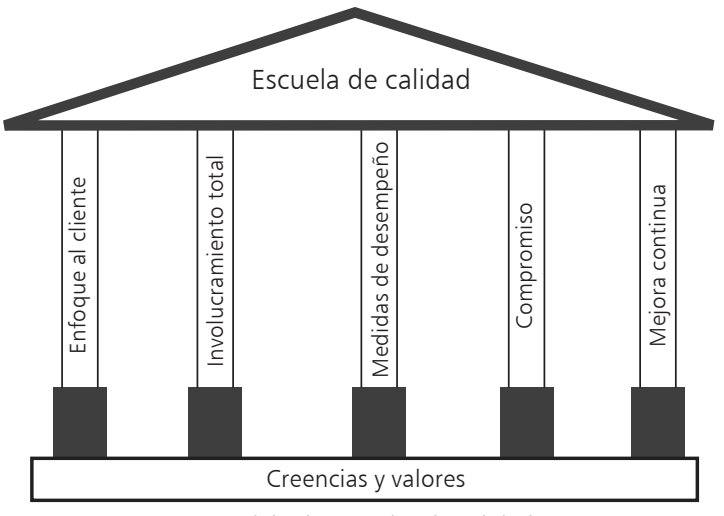

Modelo de escuelas de calidad

Fuente: Arcaro (1995, p. 28)

El modelo de gestión de calidad educativa de Arcaro se compone de cinco pilares básicos: a) Orientación al cliente; b) Involucramiento total de toda la institución; c) Establecimiento de medidas de desempeño; d) Compromiso de la Administración, y e) Implementación del programa de mejora continua en todos los indicadores señalados como prioritarios.

Arcaro describe su propio modelo de gestión de la calidad educativa de ocho puntos y refleja la influencia de los autores clásicos, ya que utiliza un círculo concéntrico de elementos con los que intenta representar la continuidad del proceso de gestión como estos recomiendan. El círculo (Figura 2) se compone de ocho pasos, con un inicio y un final, pero se reinicia periódica e interminablemente.

Figura 2.

Gestión de la calidad educativa de J. S. Arcaro

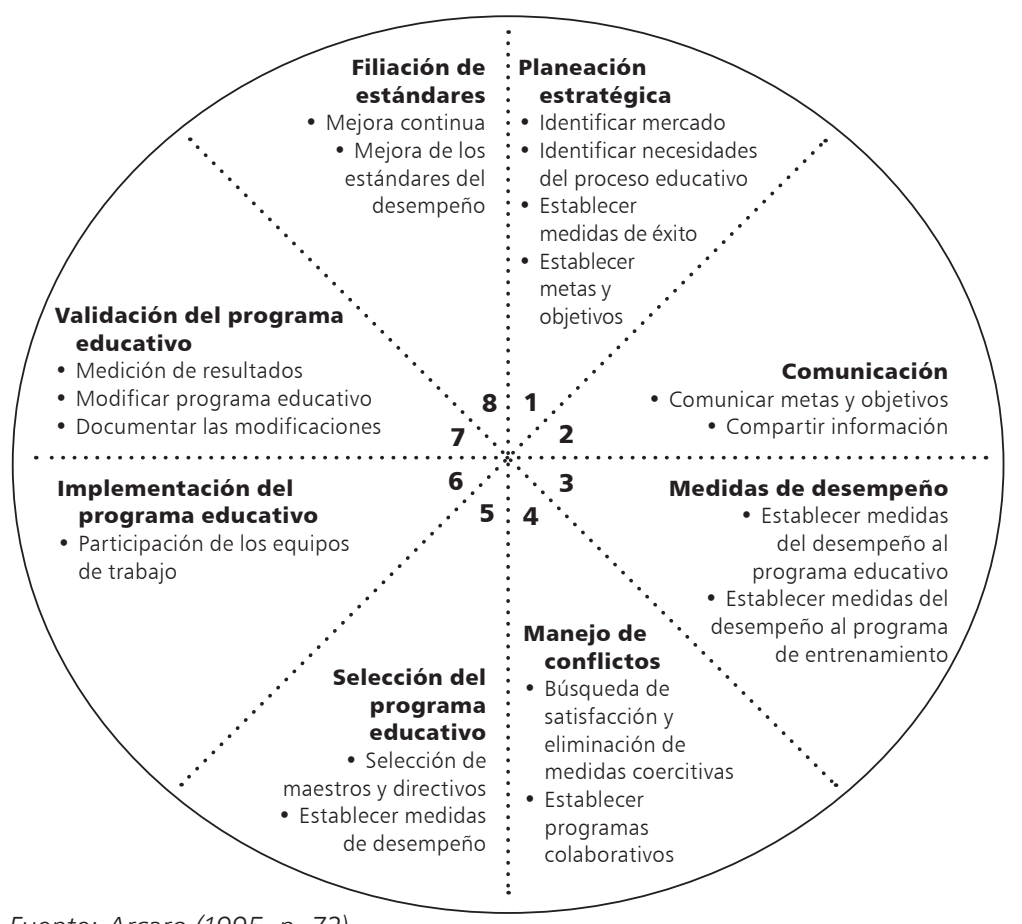

Fuente: Arcaro (1995, p. 72) 
El modelo de Arcaro parte de un proceso profundo de: (1) Planificación estratégica, apoyada en un sólido esfuerzo de (2) comunicación dentro de todos los departamentos de la organización, así como del personal y establecer, con trabajo en equipo, las diferentes (3) medidas de desempeño que se pretenden alcanzar, negociadas y aceptadas por toda la organización para evitar (4) conflictos interdepartamentales, con el fin de que el equipo de docentes y Administración seleccionen el (5) programa educativo que los llevará a la consecución de las metas establecidas y que les permita definir las estrategias necesarias para la (6) implementación del mismo, apoyado por toda la organización y la instauración paralela de las medidas de evaluación periódica de su desempeño, para obtener una clara (7) validación del programa educativo, hacer las correcciones y los ajustes necesarios y fijar los nuevos estándares de desempeño que señalará la institución educativa.

Factores y subfactores clave de éxito en la gestión de la calidad educativa Más adelante, Arcaro (1995) propone lo que llama factores clave de éxito para lograr este proceso de gestión de calidad educativa:

Tabla 2.

Factores clave de éxito según Arcaro

\begin{tabular}{|c|c|c|c|c|c|}
\hline $\begin{array}{l}\text { 1. Liderazgo de la } \\
\text { Administración }\end{array}$ & $\begin{array}{l}\text { a) Hacia el personal } \\
\text { administrativo }\end{array}$ & $\begin{array}{l}\text { b) Hacia los } \\
\text { maestros }\end{array}$ & $\begin{array}{l}\text { c) Hacia los padres } \\
\text { de los alumnos }\end{array}$ & $\begin{array}{l}\text { d) Hacia } \\
\text { la sociedad }\end{array}$ & \\
\hline $\begin{array}{l}\text { 2. Diseño } \\
\text { de las instalaciones }\end{array}$ & a) Hacia las aulas & $\begin{array}{l}\text { b) Hacia la } \\
\text { biblioteca }\end{array}$ & $\begin{array}{l}\text { c) En las } \\
\text { instalaciones } \\
\text { de apoyo } \\
\text { administrativo }\end{array}$ & & \\
\hline $\begin{array}{l}\text { 4. Implementación } \\
\text { de sistemas de } \\
\text { evaluación del } \\
\text { desempeño }\end{array}$ & a) A los maestros & b) A los alumnos & $\begin{array}{l}\text { c) Al personal } \\
\text { administrativo }\end{array}$ & $\begin{array}{l}\text { d) A los } \\
\text { programas } \\
\text { educativos }\end{array}$ & $\begin{array}{l}\text { e) A la gestión } \\
\text { escolar }\end{array}$ \\
\hline 6. Alumnos & a) Pruebas de selección & $\begin{array}{l}\text { b) Evaluación } \\
\text { del desempeño } \\
\text { académico }\end{array}$ & & & \\
\hline $\begin{array}{l}\text { 7. Calidad de } \\
\text { maestros }\end{array}$ & a) Perfil de maestros & $\begin{array}{l}\text { b) Actitud } \\
\text { de maestros }\end{array}$ & $\begin{array}{l}\text { c) Desempeño } \\
\text { didáctico }\end{array}$ & & \\
\hline $\begin{array}{l}\text { Regreso al } 1 \text { para la } \\
\text { mejora continua }\end{array}$ & $\begin{array}{l}\text { Volver a empezar } \\
\text { el ciclo }\end{array}$ & & & & \\
\hline
\end{tabular}

Fuente: Arcaro (1995)

Como se puede apreciar, el modelo de factores y subfactores clave de éxito para el aseguramiento de la calidad de Arcaro (1995) es bastante amplio e incluye siete factores y veinte subfactores de apoyo en la gestión de la calidad educativa. Por esta razón, se tomó este modelo como guía inicial para este estudio, en el entendido de que nuestros hallazgos pueden ser iguales o diferentes al modelo de Arcaro.

Alcanzar la calidad educativa es un trabajo arduo que requiere compromiso para el logro de la excelencia; implica un liderazgo dedicado a la obtención de los resultados y, sobre todo, la convicción de la necesidad de lograr un cambio de actitud en todo el personal de la institución. Los 
factores clave no funcionan en forma aislada o individual, pues su éxito depende de la articulación.

\section{Metodología}

El propósito de la investigación es determinar cuáles son los factores y subfactores para mejorar la calidad educativa y generar éxito que, desde la perspectiva de sus líderes, son utilizados en los programas de gestión de calidad educativa de instituciones de educación superior del sector privado en el estado de Nuevo León (México). Un estudio de esta naturaleza permitirá conocer y difundir aquellas herramientas de la gestión de la calidad que han sido útiles en su aplicación en instituciones de educación superior del sector privado y que podrían ser líneas de acción para aquellas instituciones educativas que aspiren a la mejora continua de sus programas educativos y que busquen, asimismo, estándares de alta calidad que les posibiliten mantenerse y figurar en el mercado de opciones educativas de nivel superior.

En este caso particular, se indaga lo que los expertos consultados definen como factores de éxito en la gestión de la calidad educativa de las IES del sector privado en el estado de Nuevo León y tratamos de definir sus respectivos subfactores de apoyo.

Aunque en este estudio se utilizó el método Delphi, bien puede decirse que los resultados que se presentan fueron obtenidos mediante entrevistas a profundidad, dado que en este artículo se reportan los resultados del primer paso en el proceso de aplicación del método Delphi: las entrevistas a profundidad. Este método permite estructurar los procesos de comunicación de un grupo de personas, de manera que sean efectivos y le facilite llegar a un consenso frente a un problema con alto grado de dificultad (Linstone \& Turoff, 1975; Clayton, 1997). El método Delphi se utiliza por varias razones:

1. Debe usarse cuando se consulta a los expertos, porque la información precisa no se encuentra disponible.

2. Es el más adecuado cuando se trata de identificar competencias.

3. Se recomienda cuando las respuestas son vagas, sujetas a muchas interpretaciones $y$, por lo tanto, es necesario ordenarlas hasta producir consenso en torno a un asunto determinado (Adler \& Ziglio, 1996).

Como lo indica la metodología Delphi, los pasos que se siguieron para realizar este estudio fueron los siguientes: a) se seleccionó al grupo de expertos con base en los criterios definidos para la muestra; b) se realizó la primera ronda de entrevistas, y c) se recopilaron y se ordenaron los datos cualitativos con el sistema NVIVO 8.

En la primera fase, se llevó a cabo una entrevista semiestructurada, que se grabó para un análisis posterior. Se inició con una pregunta abierta para pedir a los expertos que, en su opinión, detallen cuáles son los factores clave de éxito que intervienen en la gestión de la calidad educativa de las IES del estado de Nuevo León. En esta entrevista se permitió al experto expresar libre y espontáneamente sus ideas y utilizar todas las palabras, las frases y los conceptos. El investigador continuó entrevistando al líder con el fin de explorar la existencia de otros subfactores de éxito que el experto no hubiera considerado en la primera parte.

Estas entrevistas fueron examinadas con el sistema NVIVO 8, para explorar todos los factores y subfactores mencionados por los líderes en la primera ronda de entrevistas. Con este análisis se pretendía detectar cierta jerarquía entre los factores y subfactores, al considerar el total de texto empleado en la entrevista con cada líder. Los resultados de esta fase del estudio son los que se presentan en este artículo.

El sistema de análisis cualitativo NVIVO 8 es un sistema computacional que permite el análisis de datos cualitativos como discursos, entrevistas, pláticas, videos, etc., cuya función es agrupar en categorías, para su clasificación y para el análisis de la relación entre las categorías. Es posible ver los datos desde diferentes ángulos o enfoques y explorar el material recopilado de manera más ordenada para llegar así a organizar los hallazgos, encontrar respuestas y revelar significados. La forma de operación de NVIVO 8 se explica en detalle en Leech y Onwuegbuzie (2011). Todos los temas obtenidos en las respuestas de los expertos se asocian en grupos y se codifican por categoría. Mediante este procedimiento se detectaron los factores y subfactores importantes para la gestión de calidad educativa presentes en el discurso de los líderes consultados.

\section{La muestra}

Para esta investigación se seleccionó una muestra de veintidós expertos con un promedio de antigüedad en años de experiencia en educación superior de 22,8 años y una desviación estándar de 9,47 años, que agrupa a la mayoría de los expertos entre un rango de años de antigüedad de 13 a 32 años y una moda de 30 años.

\section{Criterios para participar en la muestra}

1. Operar con un sistema de gestión de calidad educativa. El concepto de "gestión de calidad" es definido por Feigenbaum (1956) como un sistema eficaz para integrar los esfuerzos realizados por los diversos grupos de una organización, de modo que sea posible 
producir bienes y servicios más económicos y compatibles con la plena satisfacción de los clientes.

2. Las IES del sector privado invitadas a participar en este estudio deben implementar factores clave de éxito que utilizan e incorporan para apoyar y elevar su programa de gestión de calidad educativa, considerados como aquellos elementos o esas características imprescindibles que una institución de educación superior debe asumir eficientemente, con el fin de mantener y elevar la calidad de su sistema educativo.

3. Los expertos consultados de las IES del sector privado o instituciones seleccionadas deben estar involucrados en los esfuerzos de gestión de calidad educativa que realiza la institución donde prestan sus servicios.

4. Es muy importante reconocer que las IES invitadas no son las únicas del sector privado del estado de Nuevo León que cumplen con los requisitos de gestión de calidad educativa que buscamos como muestra para realizar el presente estudio. Por limitaciones de tiempo y delimitaciones del estudio, solo invitamos a participar a cierto número de ellas.

5. También fueron invitadas instituciones que, en cierta forma, colaboran para mejorar la gestión de calidad educativa de las IES del sector privado en el estado, como la Secretaría de Educación del estado de Nuevo León, el Gobierno municipal de Monterrey y una agencia acreditadora de calidad internacional como TUV de México y sus respectivos expertos en calidad educativa.

Criterios para el perfil de los expertos de la muestra

El perfil de los expertos seleccionados fue enmarcado en los siguientes criterios:

1. El participante deberá haber trabajado en el área de educación superior o de gestión de calidad educativa para instituciones del sector privado por cinco años, al menos.

2. El participante deberá contar con experiencia en la coordinación de proyectos relacionados con la gestión de calidad educativa de nivel superior.

3. El participante deberá contar con algún cargo directivo u honorario con impacto en la gestión de calidad educativa en alguna IES o institución seleccionada para participar en la muestra.

\section{Resultados}

El análisis toma como referencia el discurso empleado en la discusión del tópico durante la entrevista a cada líder y el número de veces que el tema es mencionado en ellas, pues se asume que el grado de discusión de un tema indica cierto grado de preocupación y preponderancia. En esta etapa del estudio fue posible establecer todas las categorías o subcategorías, es decir, los factores y subfactores que los expertos consideran importantes para la implementación de la gestión de la calidad educativa en los programas de educación superior. Este examen y sus resultados contribuirán a la revisión y actualización del modelo de Arcaro (1995) con el que iniciamos. Estos resultados constituyen un mapa general que puede servir de guía para quienes estén interesados en los procesos de aseguramiento de la calidad de base empresarial utilizados en instituciones de educación superior en el sector privado en México y en América Latina. 
En la Tabla 3 se muestran los resultados que indican la frecuencia con que se mencionan las categorías y subcategorías (factores y subfactores) necesarias en la gestión para la calidad educativa en el nivel superior.

Tabla 3.

Factores y ponderación por número de comentarios

\begin{tabular}{lcccc}
\hline \multicolumn{1}{c}{ Categorías } & Expertos & $\%$ de expertos & $\begin{array}{c}\text { Frecuencia } \\
\text { de mención }\end{array}$ & \% de menciones \\
\hline Gestión escolar & 22 & $100,00 \%$ & 172 & $26,06 \%$ \\
\hline Diseño curricular & 20 & $90,91 \%$ & 93 & $14,09 \%$ \\
\hline Modelo educativo & 21 & $95,45 \%$ & 79 & $11,97 \%$ \\
\hline Infraestructura & 19 & $86,36 \%$ & 74 & $11,21 \%$ \\
\hline Maestros & 20 & $90,91 \%$ & 70 & $10,61 \%$ \\
\hline Planificación educativa & 20 & $90,91 \%$ & 61 & $9,24 \%$ \\
\hline Alumnos & 19 & $86,36 \%$ & 53 & $8,03 \%$ \\
\hline Vinculación con el mercado & 19 & $86,36 \%$ & 45 & $6,82 \%$ \\
\hline Liderazgo en la institución & 6 & $27,27 \%$ & 9 & $1,36 \%$ \\
\hline Factor Gobierno del país & 2 & $9,09 \%$ & 4 & $0,61 \%$ \\
\hline
\end{tabular}

Fuente: elaboración propia

Cabe aclarar que estos resultados no fueron jerarquizados por los expertos en orden de importancia, sino que son el resultado del análisis de los temas más mencionados en las entrevistas a profundidad.

La Gráfica 1 ilustra los diez temas (categorías) y su grado de presencia en el discurso:

Gráfica 1

Categorías para la gestión de la calidad educativa presente en el discurso de los líderes

\section{Porcentaje de mediciones}

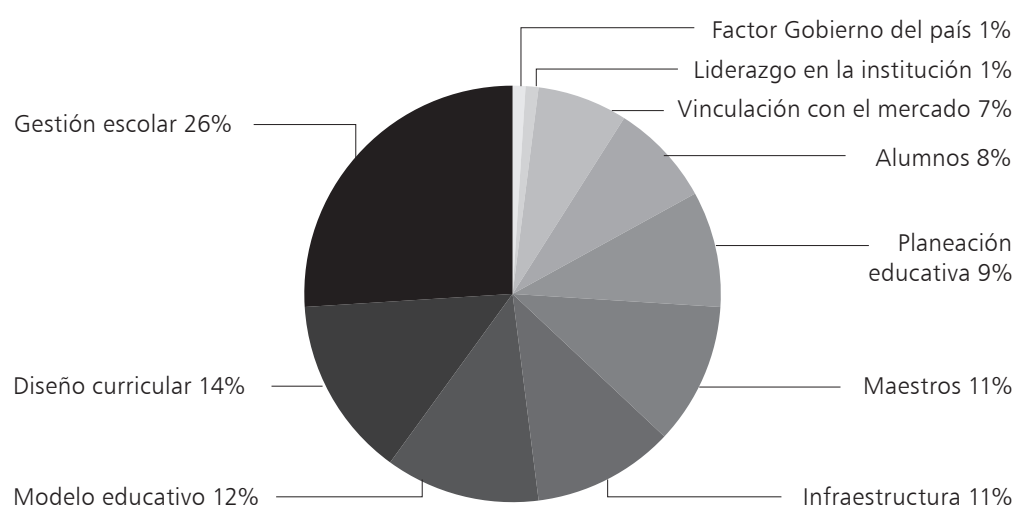




\section{Análisis de las categorías}

Gestión escolar (26\%): la discusión de los expertos se situó en primer lugar en la realización de un ejercicio institucional de análisis de fuerzas, debilidades, oportunidades y amenazas (FODA), con la finalidad de establecer los parámetros de mejora y los aspectos de sistemas de evaluación interna, en especial hacia las medidas del desempeño que cada institución seleccionó y los procesos administrativos a implementar para el avance, mediante estrategias pedagógicas y administrativas. Los expertos mencionan la importancia de seleccionar sistemas de evaluación externa que validen lo que la institución pretende optimar. En segundo lugar, la discusión se orienta a definir su tablero de indicadores del desempeño y control, en donde se identifican y se expresan estas medidas clave que muestran el avance en el desempeño de la institución para optimizar sus estándares de gestión de calidad educativa. También se plantea la necesidad de realizar ejercicios de comparación de estándares (benchmarking) con otras instituciones de educación superior, con el fin de adoptar y adaptar prácticas exitosas. Por último, debaten la importancia de formar consejos directivos en la institución, para asegurar la pertinencia de sus programas educativos e instaurar programas de tutoría a sus alumnos para apoyar su éxito educativo. Uno de los expertos expone:

L18: tú como institución no solo debes de ver lo que pasa hacia adentro de tu organización y ver tus fuerzas y debilidades; también es importante ver hacia afuera y evaluar tus oportunidades y amenazas. Tienes que ir reenfocando tu rumbo educativo constantemente.

Diseño curricular (14\%): es claro que el diseño mismo de los planes de estudio en relación con la pertinencia y actualidad fueron los aspectos en donde se concentró la discusión de los expertos, con lo que las actividades de formación integral en sus alumnos pasaron a un segundo término. Cuando los expertos comentaron sus ideas sobre cuál diseño curricular (en conexión con el modelo educativo) deberían emplear dadas las circunstancias actuales de un mundo de globalización y altos estándares de calidad, comentaron que lo que ellos proponen es que los planes de estudio se orienten a que los jóvenes salgan preparados para insertarse en el mercado laboral de cualquier parte del mundo. Esto implica que los planes de estudios de su particular institución deben de ser reconocidos por otros establecimientos pares internacionales, a tal grado que pueda hablarse de planes de estudio con doble titulación o que los alumnos estén habilitados para tomar ciertas clases en el extranjero y revalidar sus estudios. También mencionan que saber investigar y resolver problemas es una parte que los planes de estudio deben incluir. Estos son algunos de los cometarios respecto al diseño curricular: "L1: le puedo confirmar que el diseño curricular para cada una de las carreras es sustantivo, porque se tiene que vincular la preparación del nuevo profesionista con las necesidades reales del mercado laboral".

Modelo educativo (12\%): seleccionar el modelo educativo que se adapte más a los objetivos académicos fue uno de los tópicos más discutidos por los expertos, así como el enfoque hacia la investigación y la globalización de sus programas académicos con todo lo que ello implica. En relación con el tamaño de la institución, opinan que existen dos caminos para ser reconocidos como una institución de calidad educativa. Si se decide ser una institución pequeña, deberá especializarse en algo, por ejemplo, la Escuela Libre de Derecho se especializa solo en esa área o el Centro de Diseño de Monterrey se enfoca en la rama del diseño, pero sus programas son tan reconocidos, que sus egresados son aceptados en el ámbito local y global. De otro lado, si se decide ser una institución grande, hay que tener tantos programas o carreras como las universidades públicas y las grandes universidades y luchar por ser tan competitivos como aquellas. El siguiente es un ejemplo textual sobre el tema:

L4: diría que otro aspecto muy importante es el desarrollo del modelo educativo de la institución. Las instituciones pueden tener diferentes modelos educativos; sin embargo, todas ellas buscan la excelencia académica y, sobre todo, preparar a los jóvenes para que puedan ser útiles en el mercado laboral. Sin embargo, las instituciones educativas lo podremos hacer de diferente forma y maneras. Entonces, el modelo educativo es el que define icuáles son las características y materiales que tendré en las aulas? Sobre todo, ¿cuáles son las características que tendrán mis egresados?

Infraestructura (11\%): la discusión de los expertos se centró en describir prioritariamente aspectos importantes requeridos por las instalaciones. Insistieron en resaltar la creación de una buena imagen institucional para apoyar los esfuerzos de gestión de calidad educativa y mencionaron elementos importantes para formar una biblioteca a la altura de las necesidades de un programa de educación superior. En su propia voz:

L1: La biblioteca, como parte de la infraestructura de la universidad, debe comprender documentos físicos o virtuales que permitan a los estudiantes consultar cualquier tipo de duda que tengan durante los estudios de su carrera profesional. 
L5: Al hablar de infraestructura, hablamos de los salones de clases, pero también del resto de las instalaciones como lo es la biblioteca y hay que hablar de accesos a la información: vamos a decir que la infraestructura permita que los alumnos puedan acceder a los lugares o sitios mejor informados del mundo.

Maestros (11\%): los expertos discutieron la necesidad de encontrar maestros con el perfil requerido por los programas de educación superior como el tema más importante a tratar en esta categoría y pasaron a debatir, en menor medida, la importancia de mantener la actualización del personal docente y la capacitación continua.

L2: una parte muy importante debe ser la calidad y el perfil de los maestros que están dando las diferentes cátedras. ¿Qué quiero decir con esto? Que no se trata de seleccionar puros doctores para impartir los cursos, sino más bien, seleccionar al mejor maestro para dar cada curso, en base a su perfil de conocimientos y habilidades didácticas que posea para esa cátedra.

Planificación educativa (9\%): el factor de Planificación educativa ocupó la atención de los expertos como determinante en la gestión de la calidad de las IES del sector privado en el estado de Nuevo León, en busca de la adecuación al segmento socioeconómico objetivo y la relación del análisis costo-beneficio como tema importante de su agenda. La visión del cliente es vital para centrar los esfuerzos de la gestión de calidad en forma eficaz y efectiva. Dentro de lo que se categoriza como Planificación educativa se encuentran los subfactores de Planificación estratégica; Adecuación al segmento socio-económico; Visión del cliente y Relación costo-beneficio. Los expertos argumentaron que la Planificación estratégica es un subfactor importante en la gestión de calidad educativa, pero siempre y cuando esta planificación no pierda de vista el segmento económico al que prestarán sus servicios educativos, para manejar muy bien la adecuación de los costos de sus colegiaturas en relación con los beneficios adquiridos por los alumnos. Al pensar en el cliente y en cómo facilitar su acceso a la educación privada, esto es lo que opinan:

L6: Es importante para el país no solo que la calidad en la educación sea la adecuada, sino que pueda ser accesible la educación para todas las personas de los diferentes niveles sociales.

L7: Que la planeación educativa permita al país ir mejorando y no se convierta la educación superior solo en privilegio de unos cuantos que disponen de altos ingresos.
L7: Lo importante es lograr que la educación superior privada y la educación superior pública tengan el mismo nivel en calidad educativa.

Alumnos (8\%): los expertos hablaron de la importancia de que los alumnos se asemejen al perfil de ingreso que las instituciones definen para cada programa educativo de nivel superior y enseguida mencionan la preparación e implementación de programas de capacitación o bien de cursos de apoyo, con el fin de elevar y preparar a los alumnos de primer ingreso para alcanzar los estándares educativos de la institución y garantizar la eficacia didáctica de sus programas educativos. Respecto al alumno como portador de la calidad, opinan:

L9: la educación es la única actividad productiva en la que la calidad va directamente relacionada con el producto en sí mismo. ¿A qué me refiero? Me refiero a que una cosa es la percepción de calidad que tiene el estudiante cuando es cliente de la institución educativa y otra percepción es cuando en realidad estamos hablando de que es el estudiante "en sí mismo" el portador de la calidad educativa que difunde la institución, de tal suerte que es mediante su buen juicio, sus habilidades, sus conocimientos, sus actitudes con los que el estudiante va a interactuar en la sociedad y mediante los cuales va a demostrar si hubo o no hubo una calidad educativa como producto final por parte de la institución que lo capacitó.

Vinculación con el mercado (7\%): el aspecto más abierto a discusión por los expertos en cuanto a este aspecto es el establecimiento de bolsas de trabajo para propiciar oportunidades de empleos tanto a los egresados como a los estudiantes. Asimismo, oportunidades para realizar sus prácticas profesionales, con el fin de garantizar una conexión entre los conocimientos teóricos aprendidos en las universidades y las oportunidades de ponerlos en práctica. Formar una incubadora de negocios que apoye el espíritu emprendedor en sus alumnos, los programas de educación continua y talleres para la comunidad son también subfactores clave de éxito en la gestión de la calidad educativa en este factor. Afirman:

L13: creemos que la responsabilidad de una universidad no termina cuando se gradúa uno de sus alumnos. El vínculo universidad-alumno-mercado no termina jamás, ya que el lazo de comunicación permanece al apoyar la institución [para] que el alumno encuentre un empleo y continúa cuando se da seguimiento y desarrollo a su carrera profesional, de tal suerte que la institución apoya al alumno 
a descollarse profesionalmente y el alumno retroalimenta a la universidad en cómo lograr que se abran los caminos para sus egresados y qué factores le recomendará a su universidad, para que siga perfeccionando sus planes de estudio y se adecúen a las necesidades del mercado.

Liderazgo en la institución (1\%): el liderazgo del rector fue un tema muy discutido por los asesores como factor importante en la gestión de calidad educativa y, en menor medida, el liderazgo del fundador o fundadores de la institución. Opinan:

L6: cuando una institución educativa tiene un buen líder, que quiere hacer que las cosas funcionen, aun cuando existan ciertas restricciones en diferentes factores clave como lo son la calidad y preparación de los maestros, la calidad de las instalaciones, la calidad de los materiales educativos, [etc.], el ser buen líder te posibilita sacar el mejor provecho de los recursos existentes y esto te permite acercarte a obtener buenos resultados didácticos como institución, así como mejorar los enlaces con tu comunidad [y] las relaciones con tus maestros y alumnos; mejoran muchos otros factores, porque no solamente es importante la labor de los maestros, sino la labor del líder de la institución, con el fin de entender las necesidades de su comunidad, sus alumnos, sus padres de familia y el mercado laboral.

Factor Gobierno del país (1\%): aquí se centró la discusión de los expertos en señalar la importancia de ajustarse a las normativas que exigen las leyes de educación, es decir, la Ley Federal de Educación y la Ley Estatal de Educación con sus respectivos reglamentos. Además, mostraron preocupación por el manejo del presupuesto en educación:

L8: un factor muy importante tiene que ver con los fondos que destina el Gobierno de un país para apoyar los planes educativos de nivel superior. Me parece que muchos países tienen sus limitaciones que derivan principalmente de la cuestión presupuestaria, es decir, en función de cuantos [más] fondos del presupuesto de egresos se destinen al gasto educativo de nivel superior, se presume que tendrán una mejor cobertura y calidad en sus programas.

L9: Es importante el gasto en educación, pero es más importante analizar la manera en cómo se va a realizar ese gasto de dinero, es decir, analizar: ¿Para qué se está invirtiendo? ¿En qué se está invirtiendo? ¿Cómo se está invirtiendo? y finalmente ver si se cuenta con un plan estratégico a seguir por parte del Gobierno.

Con este análisis, es posible utilizar el modelo de Arcaro para revisar si hay elementos emergentes en el discurso de los expertos en relación con los factores y subfactores críticos para la gestión de la calidad en educación superior.

\section{Actualización del modelo de Arcaro para la gestión de la calidad educativa: elementos emergentes}

A partir del análisis por categorías, se puede regresar a los factores y subfactores propuestos por Arcaro (1995), para intentar una comparación que nos permita actualizar el modelo y hacerlo más pertinente para un nuevo contexto, un nuevo período y con nuevos especialistas que, en la práctica cotidiana, operan y gobiernan instituciones de educación superior del sector privado y consideran los factores que influyen en la calidad y 
en el éxito de sus instituciones. El modelo de Arcaro (1995) funciona como base sobre la cual fue posible avanzar para agregar y aportar elementos que surgen en este estudio en las voces de los líderes de la muestra. En la siguiente sección se analiza cada una de las categorías mencionadas por los expertos y presentadas en la Tabla 4. Estas categorías son el resultado del análisis de los tópicos más mencionados en las entrevistas a profundidad. Asimismo, se presenta la comparación con el modelo de Arcaro y se discuten las semejanzas y diferencias entre este modelo y la teoría emergente en este estudio.

Tabla 4.

Comparación entre los factores del modelo de Arcaro (1995) y los factores enunciados por la muestra de líderes entrevistados en el estado de Nuevo León

\begin{tabular}{|c|c|c|}
\hline Modelo de Arcaro & Modelo emergente & Comentarios \\
\hline $\begin{array}{l}\text { 1. Liderazgo de la } \\
\text { Administración } \\
\text { a) Hacia el personal } \\
\text { administrativo } \\
\text { b) Hacia los maestros } \\
\text { c) Hacia los padres de los } \\
\text { alumnos } \\
\text { d) Hacia la sociedad }\end{array}$ & $\begin{array}{l}\text { 1. Liderazgo en la institución } \\
\text { a) Liderazgo del rector } \\
\text { b) Liderazgo del fundador o de los } \\
\text { fundadores }\end{array}$ & $\begin{array}{l}\text { Aquí los expertos hablan de un liderazgo hacia } \\
\text { los nueve aspectos importantes de la gestión } \\
\text { de calidad (Planificación educativa; Modelo } \\
\text { educativo; Gestión escolar; Diseño curricular; } \\
\text { Infraestructura; Perfil de maestros; Perfil de } \\
\text { alumnos; Vinculación con mercado; y Factor } \\
\text { Gobierno) mientras que Arcaro solo los enfo- } \\
\text { ca a cuatro aspectos (personal administrativo, } \\
\text { docentes, padres de alumnos y sociedad). }\end{array}$ \\
\hline $\begin{array}{l}\text { 2. Diseño de las instalaciones } \\
\text { a) Hacia las aulas de clases } \\
\text { b) Hacia la biblioteca } \\
\text { c) En las instalaciones de } \\
\text { apoyo administrativo }\end{array}$ & $\begin{array}{l}\text { 2. Infraestructura } \\
\text { a) Hacia instalaciones } \\
\text { b) Hacia la biblioteca } \\
\text { c) Hacia la imagen institucional }\end{array}$ & $\begin{array}{l}\text { Los expertos direccionan la importancia de las } \\
\text { instalaciones en su conjunto y orientadas a } \\
\text { elevar siempre la imagen institucional. Arcaro } \\
\text { solo señala aulas de clases, biblioteca y áreas } \\
\text { administrativas. }\end{array}$ \\
\hline $\begin{array}{l}\text { 3. Diseño curricular } \\
\text { a) Adaptado a las } \\
\text { necesidades de la sociedad } \\
\text { b) Actualizado }\end{array}$ & $\begin{array}{l}\text { 3. Diseño curricular } \\
\text { a) Planes de estudio pertinentes } \\
\text { b) Actividades de formación integral }\end{array}$ & $\begin{array}{l}\text { La gran diferencia es que los expertos resal- } \\
\text { tan la importancia de la educación integral del } \\
\text { individuo y su adaptación a la globalización. } \\
\text { Arcaro solo indica como importante la perti- } \\
\text { nencia de los planes de estudio. }\end{array}$ \\
\hline $\begin{array}{l}\text { 4. Sistemas de evaluación del } \\
\text { desempeño } \\
\text { a) Para maestros } \\
\text { b) Para alumnos } \\
\text { c) Al personal administrativo } \\
\text { d) A los programas } \\
\text { educativos } \\
\text { e) A la gestión escolar }\end{array}$ & $\begin{array}{l}\text { 4. Gestión escolar eficiente } \\
\text { a) Procesos administrativos eficientes } \\
\text { b) Implementar sistemas de } \\
\text { evaluación interna } \\
\text { c) Implementar sistemas de } \\
\text { evaluación externa } \\
\text { d) Realizar un análisis FODA } \\
\text { e) Realizar ejercicios de benchmarking } \\
\text { (comparación de estándares entre } \\
\text { instituciones similares) } \\
\text { f) Establecer un tablero control } \\
\text { g) Formar consejos directivos } \\
\text { h) Establecer programas de tutoría }\end{array}$ & $\begin{array}{l}\text { Los puntos } 4 \text { y } 5 \text { de Arcaro se engloban en } \\
\text { el punto } 4 \text { señalado por los expertos. Arcaro } \\
\text { solo habla de evaluación y los expertos, de fi- } \\
\text { jar metas y objetivos y después medir avances } \\
\text { y evaluarlos. }\end{array}$ \\
\hline $\begin{array}{l}\text { 5. Gestión escolar } \\
\text { a) A las prácticas } \\
\text { administrativas } \\
\text { (benchmarking) }\end{array}$ & $\begin{array}{l}\text { 5. Planificación educativa } \\
\text { a) Planificación estratégica } \\
\text { b) Adecuación al segmento socio- } \\
\text { económico meta } \\
\text { c) Visión de cliente } \\
\text { d) Relación costo-beneficio }\end{array}$ & $\begin{array}{l}\text { El elemento emergente es la adecuación de } \\
\text { la planificación educativa en función del seg- } \\
\text { mento de población a quien va dirigido y de la } \\
\text { relación costo-beneficio. }\end{array}$ \\
\hline $\begin{array}{l}\text { 6. Alumnos } \\
\text { a) Pruebas de selección } \\
\text { b) Evaluación del } \\
\text { desempeño académico }\end{array}$ & $\begin{array}{l}\text { 6. Alumnos. } \\
\text { a) Perfil de ingreso } \\
\text { b) Capacitación a alumnos (cursos de } \\
\text { apoyo para superar problemas) }\end{array}$ & $\begin{array}{l}\text { Arcaro incluye las pruebas de selección y la } \\
\text { evaluación del desempeño académico, mien- } \\
\text { tras que los expertos entrevistados agregan } \\
\text { los cursos de apoyo para ayudar a los estu- } \\
\text { diantes a lograr el perfil deseado de ingreso } \\
\text { y de acompañamiento durante sus estudios } \\
\text { para asegurar el perfil deseado de egreso. }\end{array}$ \\
\hline $\begin{array}{l}\text { 7. Calidad de los maestros } \\
\text { a) Perfil de los maestros } \\
\text { b) Actitud de maestros } \\
\text { c) Desempeño didáctico }\end{array}$ & $\begin{array}{l}\text { 7. Maestros } \\
\text { a) Perfil de los maestros } \\
\text { b) Capacitación de maestros }\end{array}$ & $\begin{array}{l}\text { Las actitudes docentes son un elemento im- } \\
\text { portante en Arcaro que no está presente en el } \\
\text { discurso aquí analizado. }\end{array}$ \\
\hline
\end{tabular}




\begin{tabular}{|c|c|c|}
\hline Modelo de Arcaro & Modelo emergente & Comentarios \\
\hline
\end{tabular}

Fuente: elaboración propia

\section{Conclusiones}

El propósito de este artículo fue indagar sobre los factores que influyen en el éxito de las instituciones de educación superior por medio de entrevistas a profundidad con una muestra de veintidós líderes de dichas instituciones. Un análisis cualitativo de sus opiniones en dichas entrevistas, con la utilización del programa NVIVO 8, permitió detectar algunas diferencias, por lo que emergen algunos factores nuevos.

El examen parece mostrar coincidencias entre los primeros siete factores propuestos por Arcaro: 1. Liderazgo de la Administración; 2. Diseño de las instalaciones; 3. Diseño curricular; 4. Implementación de sistemas de evaluación del desempeño; 5. Gestión escolar; 6. Alumnos, y 7. Calidad de maestros, así como mínimas discrepancias en estos factores y sus subfactores, que fueron explicadas en la Tabla 4.

Sin embargo, más allá de estos siete factores, este estudio parece mostrar otros emergentes que reflejan el contexto de mercado y de competencia propios de la globalización de este siglo.

Los factores emergentes son tres: el modelo educativo; la vinculación con el mercado y factor Gobierno del país, que despliegan los siguientes subfactores:

Tabla 5.

Factores clave de éxito en la gestión de la calidad educativa emergentes en este estudio
8. Modelo educativo
9. Vinculación con el mercado
10. Factor Gobierno del país

\begin{tabular}{lll}
\hline Subfactores & Subfactores & Subfactores \\
\hline a) Modelo de plan académico & a) Programa para emprendimiento & a) Cumplir con la Ley Federal de Educación \\
b) Enfoque a la investigación & b) Bolsa de trabajo & b) Cumplir con la Ley Estatal de Educación \\
c) Tamaño de la institución & c) Educación continua & c) Cumplir con el Reglamento de la \\
d) Enfoque a la globalización & d) Talleres a la comunidad & Secretaría de Educación del estado de \\
& & Nuevo León \\
& d) Cumplir con las observaciones y \\
& recomendaciones en las inspecciones de la \\
& Secretaría de Educación del estado
\end{tabular}

Fuente: elaboración propia 
Lo que aquí se puede ver en el factor emergente referente al modelo educativo es su estrecha relación con el mercado laboral, en la que el Modelo educativo debe garantizar el empleo de los recursos humanos egresados de las instituciones como fuerza laboral. No parecen avistar ningún otro propósito para la educación superior que no sea el mejorar la empleabilidad de sus egresados. El enfoque en la investigación también parece estar orientado a encontrar soluciones para el mercado y la industria, en un afán de crear más vínculos con el mercado laboral en beneficio de su alumnado. ¿Y dónde queda la búsqueda de la verdad como afán universitario?

Sobre la Vinculación con el mercado, esta hace patente la actual y estrecha relación escuela-empresa que tienen las universidades del sector privado que, con la influencia de la globalización, permiten que el mercado laboral, nacional o extranjero, adquiera mano de obra calificada y capacitada con recursos de países en desarrollo como México, para su utilización en la industria mexicana o más allá de las fronteras. En las bolsas de trabajo participan empresas extranjeras que contratan a los mejores perfiles para trabajar en el extranjero, lo que nos obliga a pensar en cómo vamos a retener el talento nacional.

En el Factor Gobierno del país, se mencionan las preocupaciones por cumplir con leyes y reglamentos oficiales sobre educación. En este rubro, existen proyectos gubernamentales que impulsan la relación escuela-empresa para brindar soluciones científicas y tecnológicas a diferentes sectores, incluidos los sociales. Una iniciativa polémica del presidente saliente constituyó en otorgar deducibilidad fiscal en las colegiaturas de la educación privada y becas para permitir mayor acceso a la educación superior privada. El país se inserta de lleno en esta corriente global mientras líderes de la educación pública aseguran que con la mitad de dicho financiamiento se podría extender la cobertura de la educación pública. Es necesario seguir analizando estas tendencias y vigilar que tanto la educación pública como la privada contribuyan al desarrollo del país con programas de buena calidad.

Con estos hallazgos sería pertinente continuar investigando para determinar el grado de ponderación que los expertos asignan a los diez factores y 35 subfactores reportados en este artículo, para encontrar la clave exacta del éxito en la gestión de la calidad educativa en el nivel superior.

Es necesario seguir investigando los rumbos que están siguiendo tanto las universidades privadas como las públicas, con la finalidad de mejorar la calidad educativa de sus egresados y acortar distancias con la homologación de planes de estudio y mayor inclusión, en beneficio de una sociedad nacional y mundial que anhela superar su calidad de vida o salir de su pobreza, a sabiendas de que el único camino que existe para lograrlo es la educación integral.

\section{Sobre los autores}

Martha Armida Fabela-Cárdenas es doctora en Educación (PhD), Universidad de Manchester (Inglaterra). Magíster en Educational Technology and English Language Teaching, de esa misma Universidad. Magíster en Formación y Capacitación de Recursos Humanos, Universidad Autónoma de Nuevo León (UANL). Licenciada en Traducción de Inglés por la UANL. Ha llevado a cabo varias investigaciones sobre actitudes de docentes y estudiantes frente al cambio educativo, la formación de los docentes de lenguas y las teorías implícitas de los docentes frente a niños con necesidades educativas especiales, entre otras. Es profesora investigadora en el posgrado de la Facultad de Filosofía y Letras de la UANL, en donde dirige tesis de maestría y de doctorado. Es miembro nivel 1 del Sistema Nacional de Investigadores del Consejo Nacional de Ciencia y Tecnología. 
Alfonso Hernán García-Treviño es licenciado en Economía, Instituto Tecnológico y de Estudios Superiores de Monterrey. Doctor en Educación, Universidad Autónoma de Nuevo León; magíster en Administración de Empresas, del Instituto Tecnológico y de Estudios Superiores de Monterrey (ITESM). Magíster en Economía, Universidad Autónoma de Nuevo León. Se ha desempeñado como investigador en la Universidad Regiomontana y como director del Departamento de Educación Superior de la Secretaría de Educación del estado de Nuevo León. En la actualidad es consultor en temas de administración de la calidad en instituciones de educación superior de Nuevo León.

\section{Referencias}

Adler, M., \& Zigilio, E. (eds.) (1996). Gazing into the Oracle: The Delphi Method and its Application to Social Policy and Public Health. Londres: Jessica Kingsley Publishers.

Arcaro, J. S. (1995). Quality in Education: An Implementation Handbook. Delray Beach: St. Lucie Press.

Asociación Nacional de Universidades e Instituciones de Educación Superior [Anuies]. (2013). Confluencia: Noticias de la ANUIES: ISO 9000. Recuperado de www.anuies.mx

Clayton, M. (1997). Delphi: A Technique to Harness Expert Opinion for Critical Decision-Making Tasks in Education. Educational Psychology, 17 (4), 373-386.

Deming, W. E. (1994). The New Economics in Industry, Government and Education, 2a ed. Cambridge: MIT.

Feigenbaum, A. V. (1956). Total Quality Control. Harvard Business Review, 34 (6), 94-98.

Furst-Bowe, J. A., \& Bauer, R. A. (2007). Application of the Baldrige Model for Innovation in Higher Education. New Directions for Higher Education, (137). Recuperado de http://tccl.rit.albany.edu/ knilt/images/0/02/Balrige_Model_for_HE.pdf

Hernández-Sampieri R., Fernández-Collado C., \& Baptista Lucio, P. (2006). Metodología de la Investigación, 4a ed. México D. F.: Mc Graw-Hill.

Ishikawa, K. (1986). ¿Qué es el control total de calidad?: La modalidad japonesa. Bogotá: Norma.

Juran, J. (2000). How to Think About Quality. En J. M. Juran \& A. B. Godfrey (eds.), Juran's Quality Handbook, 5a ed. Nueva York: McGraw-Hill International.
Laval, C. (2004). La escuela no es una empresa: El ataque neoliberal a la enseñanza pública. Barcelona: Paidós.

Leech, N. L., \& Onwuegbuzie, A. J. (2011). Beyond Constant Comparison Qualitative Data Analysis: Using NVivo. School Psychology Quarterly, 26 (1), 70-84.

Linstone, H. A. \& Turoff, M. T. (eds.) (1975). The Delphi Method Techniques and Applications. Londres: Addison-Wesley.

Peck, C., \& Reitzug, U. C. (2012). How Existing Business Management Concepts Become Schools Leadership Fashions. Educational Administration Quarterly, 48 (2), 347-381.

Rey, R., \& Santa María, J. M. (2000). Transformar la educación en un contrato de calidad. Barcelona: Wolters Kluwer Education.

Secretaría de Educación Pública. (2012). Guía para formular el Programa Integral de Fortalecimiento Institucional (PIFI) 2012-2013. Recuperado de http://pifi.sep.gob.mx/ScPIFI/GPIFI_2012/guia/1/2

Sierra, R. (1994). Técnicas de investigación social. Madrid: Paraninfo.

Stahl, N., \& Stahl, R. (1991). We Can Agree After All! Achieving Consensus for a Critical Thinking Component of a Gifted Program Using the Delphi Technique. Roeper Review, 14 (2), 79-88.

Steed, C., Maslow, D., \& Mazaletskaya, A. (2005). The EFQM Excellence Model for Deploying Quality Management: A British-Russian Journey. Higher Education in Europe, 30 (3-4), 307-319.

Temple, P. (2005). The EFQM Excellence Model ${ }^{\circledR}$ : Higher Education's Latest Management Fad? Higher Education Quarterly, 59 (4), 261-274.

Unesco. (2006). Directrices en materia de calidad de la educación superior a través de las fronteras. Recuperado de http://unesdoc.unesco.org/ images/0014/001433/143349s.pdf

Unesco. (2007). Educación de calidad para todos: un asunto de derechos humanos. Recuperado de http://www.unesco.org.uy/educacion/fileadmin/ templates/educacion/archivos/EducaciondeCalidadparaTodos.pdf

Unesco. (2008). Reflexiones en torno a la evaluación de la calidad educativa en América Latina y el Caribe. Laboratorio latinoamericano de evaluación de la calidad en educación (Llece). Recuperado de http://unesdoc.unesco.org/images/ 0017/001776/177648s.pdf 